\title{
Optimizing Chemically Induced Resistance in Tomato Against Botrytis cinerea
}

Estrella Luna and Emily Beardon, P3 Institute for Translational Plant and Soil Biology, Department of Animal and Plant Sciences, The University of Sheffield, Sheffield, S10 2TN, UK; Sabine Ravnskov, Department of Agroecology, Aarhus University, 4200 Slagelse, Denmark; and Julie Scholes and Jurriaan Ton, P3 Institute for Translational Plant and Soil Biology, Department of Animal and Plant Sciences, The University of Sheffield

\begin{abstract}
Luna, E., Beardon, E., Ravnskov, S., Scholes, J. D., and Ton, J. 2016. Optimizing chemically induced resistance in tomato against Botrytis cinerea. Plant Dis. 100:704-710.

Resistance-inducing chemicals can offer broad-spectrum disease protection in crops, but can also affect plant growth and interactions with plantbeneficial microbes. We have evaluated different application methods of $\beta$-aminobutyric acid (BABA) and jasmonic acid (JA) for long-lasting induced resistance in tomato against Botrytis cinerea. In addition, we have studied nontarget effects on plant growth and root colonization by arbuscular mycorrhizal fungi (AMF). Germinating seeds for 1 week in BABAor JA-containing solutions promoted seed germination efficiency, did not affect plant growth, and induced resistance in 4-week-old plants. When formulating BABA and JA in carboxymethyl cellulose seed coating, only

BABA was able to induce resistance in 4-week-old plants. Root treatment of 1-week-old seedlings with BABA or JA also induced resistance in 4-week-old plants. However, this seedling treatment repressed plant growth at higher concentrations of the chemicals, which was particularly pronounced in hydroponically grown plants after BABA treatment. Both seed coating with BABA, and seedling treatments with BABA or JA, did not affect AMF root colonization in soil-grown tomato. Our study has identified commercially feasible application methods of BABA and $\mathrm{JA}$, which induce durable disease resistance in tomato without concurrent impacts on plant growth or colonization by plant-beneficial AMF.
\end{abstract}

Tomato (Solanum lycopersicum) is a major crop with a worldwide production of over 163 million tons in 2013 (FAOSTAT 2014). Tomato production is threatened by various plant diseases, including the gray mold fungus Botrytis cinerea. This necrotrophic ascomycete infects green tissues and fruit of tomato and can be controlled by application of different fungicides (Elad et al. 2007). Once the disease has been detected in green tissues, repeated applications of penthiopyradbased fungicides can significantly reduce disease by the fungus (Troisi et al. 2012). New legislation to limit fungicide usage, such as The European Union Directive 2009/128/EC, as well as evolution of fungicide resistance, have increased interest in alternative crop protection strategies that can be used in a framework of integrated pest and disease management strategies (Brévault and Bouyer 2014). Development of new disease protection strategies is essential to achieve an efficient and sustainable tomato industry.

Basal resistance of tomato against $B$. cinerea involves a wide spectrum of defense mechanisms. Apart from defenses that are regulated by the plant hormone jasmonic acid (JA) (El Oirdi et al. 2011), timely deposition of hydrogen peroxide and cell wall defenses at the sites of infection contribute to resistance against $B$. cinerea (Asselbergh et al. 2007). Like other plants, tomato can enhance its level of basal resistance after perception of specific biotic or abiotic signals. This phenomenon is commonly referred to as induced resistance. Disease protection by induced resistance can be long lasting and is often associated with a sensitization or "priming" of basal defense responses (Pastor et al. 2013).

Various chemicals have been reported to induce resistance in tomato against a wide range of pests and pathogens. For instance, salicylic acid (SA) induces resistance in tomato against Fusarium oxysporum (Mandal et al. 2009), whereas foliar applications of methyl jasmonate (MeJA) induce resistance against root-knot nematodes (Fujimoto et al. 2011) and necrotrophic pathogens (Kępczyńska and Król 2012). In addition to these plant hormones,

Corresponding author: J. Ton; E-mail: j.ton@ sheffield.ac.uk

Accepted for publication 23 September 2015.

http://dx.doi.org/10.1094/PDIS-03-15-0347-RE

(C) 2016 The American Phytopathological Society there are other chemicals that can trigger an induced resistance response in tomato. Adipic acid and amide derivatives can protect tomato against Alternaria solani (Flors et al. 2003), whereas hexanoic acid has been reported to induce resistance against $P$ seudomonas syringae pv. tomato DC3000 (Scalschi et al. 2013) and $B$. cinerea (Vicedo et al. 2009). Induced resistance by pathogenassociated molecular patterns, such the deacetylated chitin derivative chitosan (Iriti and Faoro 2009), can successfully reduce pre- and postharvest diseases in tomato (Bautista-Baños et al. 2006). Finally, the xenobiotic amino acid $\beta$-amino butyric acid (BABA) has emerged as a priming agent with an exceptionally wide range of effectiveness against tomato diseases, including biotrophic and necrotrophic pathogens (Cohen 2002; Cohen et al. 1994; Luna et al. 2014b; Oka et al. 1999).

Although most studies assess levels of induced resistance several days after induction treatment, induced resistance is often referred to as a long-lasting immune response (Durrant and Dong 2004). This durable nature offers potential to reduce repeated and energyconsuming applications of fungicides. Recently, we reported that root application of BABA to 5-day-old Arabidopsis seedlings induces resistance to (hemi)biotrophic pathogens that lasts up to 4 weeks after treatment (Luna et al. 2014a). The durability of JA-dependent resistance against herbivorous insects in both Arabidopsis and tomato can even extend into the following generation (Rasmann et al. 2012). Worrall et al. (2012) showed that seed treatment of 'Carousel' tomato with MeJA or BABA induced longlasting resistance against spider mites (Tetranychus urticae) and powdery mildew (Oidium neolycopersici), respectively, offering a relatively simple and cheap method to control pests and diseases in tomato. These findings were recently corroborated by Paudel et al. (2014), who demonstrated that seed treatment with MeJA induces long-lasting resistance against herbivory by Helicoverpa zea. However, seed treatment of tomato does not induce resistance in all tomato cultivars. Smart et al. (2013) showed that cultivar Carousel displays reduced oviposition by $T$. urticae following MeJA seed treatment, whereas this same treatment had no such effect in cultivar Money-Maker. Therefore, it seems that the effectiveness of tomato seed treatment depends on cultivar-specific variation in permeability of the seed coat.

Despite ample evidence that chemical defense activators can protect crops against diseases, they are rarely used in current agricultural practices. There are various reasons for this. First, chemically induced resistance is rarely fully effective in suppressing plant disease. 
Typically, induced resistance slows down disease progression, rather than eliminating the disease (Walters et al. 2013). Second, resistanceinducing chemicals can have negative side effects on plant growth and seed production, especially when applied in higher concentrations. These trade-offs have been attributed to the physiological costs of inducible defense mechanisms (van Hulten et al. 2006; Walters and Heil 2007). Third, resistance-inducing chemicals can affect interactions with plant-growth-promoting microorganisms, such as arbuscular mycorrhizal fungi (AMF). Although acibenzolar-S-methyl (BTH), in its commercial formulation BION, and BABA do not affect root colonization by AMF in barley and sunflower, respectively (Sonnemann et al. 2002; Tosi and Zazzerini 2000), application of BTH to soybean has been reported to reduce AMF root colonization (de Román et al. 2011). These contradictory findings highlight the need for specific case studies to assess crop-specific nontarget effects by resistance-inducing chemicals.

The objectives of this study were to (i) evaluate different application methods of BABA and JA for their ability to induce durable resistance against $B$. cinerea in tomato and (ii) assess nontarget effects of BABA and JA on seed germination, plant growth, and root colonization by AMF.

\section{Material and Methods}

Plant material and chemicals. Money-Maker tomato (S. lycopersicum) was used for all experiments. BABA (catalog number A4420-7) and JA (catalog number J2500) were obtained from Sigma-Aldrich. BABA was prepared freshly in distilled water and diluted to appropriate concentrations. Stock solutions of JA were prepared by dissolving $250 \mathrm{mg}$ in $2 \mathrm{ml}$ of ethanol, which was then diluted in distilled water to a final stock concentration of $10 \mathrm{mM}$ and kept at $-20^{\circ} \mathrm{C}$. Before usage, the $10 \mathrm{mM}$ stock solution was thawed and diluted in water to the indicated concentrations.

Seed germination analysis. Germination efficiencies of chemically treated seed were determined on the basis of average percentage of germination at different days after the start of seed imbibition $(n=$ 60 ; extended seed treatment experiments) or soil planting ( $n=12$; seed coating experiments).

Plant growth analysis. For all experiments, relative growth rates (RGR) were determined between 2 and 3 weeks after the start of the experiment (i.e., planting of seed). RGR was based on the distance between the soil surface and the apical meristem, using the formula

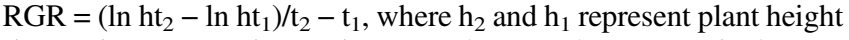
(in centimeters) at time points $t_{2}$ and $t_{1}$ (weeks), respectively. Bar graphs represent RGR means \pm standard error of the mean (SEM; $n=8$ to 10 ).

Cultivation of $B$. cinerea and inoculations. B. cinerea strain R16 (Faretra and Pollastro 1991) was cultivated on potato dextrose agar (PDA; Difco). To produce spores for plant inoculation, one agar plug ( $5 \mathrm{~mm}$ in diameter) from a fully colonized plate was placed in the center of a new PDA plate and kept in the dark at room temperature for 4 weeks. Spores were scraped from agar plates in $20 \mathrm{ml}$ of sterile water containing $0.01 \%$ Tween 20 . Spores were then filtered through Miracloth (EDM Millipore) and precipitated by centrifuging for $10 \mathrm{~min}$ at 4,000 rpm. Spores were resuspended in sterile water and adjusted to $10^{7}$ spores $/ \mathrm{ml}$. Preparation of the inoculum and the inoculation of leaves were performed, as described by Audenaert et al. (2002). Briefly, inoculum was prepared by adjusting the spore concentration to $10^{6}$ spores $/ \mathrm{ml}$ in $10 \mathrm{mM}$ glucose and $\mathrm{KH}_{2} \mathrm{PO}_{4}(\mathrm{pH} 5)$ and incubated at room temperature for $2.5 \mathrm{~h}$. In all, 5 to 10 plants per treatment were used for the infection with $B$. cinerea. For each plant, the two youngest and fully expanded leaves with at least five leaflets were selected for inoculation. Leaves were excised, after which the three biggest leaflets were inoculated by applying two $5-\mu l$ drops on either side of the main vein (i.e., 6 drops/leaf). Inoculated leaves were kept in the dark at $26^{\circ} \mathrm{C}$ and $100 \%$ relative humidity ( $\mathrm{RH}$ ) for 2 or 4 days to allow for disease progression. To account for interexperiment variation in $B$. cinerea virulence, randomly selected lesion diameters in control-treated plants were monitored on a daily basis. Scoring of the entire experiment was performed when control-treated plants had reached an average lesion diameter between 2 and $10 \mathrm{~mm}$, typically between 2 and 4 days after inoculation (dai). One biologically replicated measurement represented the average lesion diameter per plant, which was calculated from a total of 12 lesions on two different leaves. Bar graphs represent means of lesion diameters (in millimeters) $\pm \operatorname{SEM}(n=5$ to 10$)$.

Statistical analysis and software. Differences in numbers of germinated versus nongerminated seed and in distributions of inoculation sites over hyphal colonization classes were analyzed by Fisher's exact tests $(\alpha=0.01)$. For analysis of RGR and lesion diameters, normal distributions were confirmed by Shapiro-Wilk tests, whereas equality of variances was determined by Levene's tests. If equality of variances could be confirmed, differences between means were analyzed using independent-sample $t$ tests. If the Levene's test revealed unequal variances between treatments, a Welch's $t$ test was performed. Asterisks above bar graphs indicate statistically significant differences in comparison with the corresponding control treatments. A single asterisk $(*)$ indicates a statistical difference at $P<0.05<0.01$, whereas double asterisks $(* *)$ indicate a statistical difference at $P<0.01$. All analyses were conducted with IBM SPSS Statistics software (version 19.0).

Pilot experiment to validate detached-leaf assay. Tomato plants were cultivated under controlled environment conditions (cycle of 16 and $8 \mathrm{~h}$, day and night, 26 and $20^{\circ} \mathrm{C}$, respectively) in $400-\mathrm{ml}$ pots containing Scotts Levington M3 soil (Everris). Three-week-old plants were treated with water (control), BABA, or JA to a final concentration in the soil of $1 \mathrm{mM}$ BABA or $0.1 \mathrm{mM} \mathrm{JA}$. Chemical treatments were performed by injecting the soil with a $10 \times$ concentrated solution at a volume that was $10 \times$ lower than the pot volume (i.e., $40 \mathrm{ml}$ of solution into $400-\mathrm{ml}$ pots). Five days after chemical treatments, leaves from 10 different plants per treatment were detached and the five leaflets of each leaf were inoculated with $B$. cinerea. From 2 to 3 dai, 30 to 40 inoculation sites at the smallest leaflets were selected for trypan-blue staining, as described previously (Audenaert et al. 2002). Hyphal colonization by B. cinerea was assessed microscopically (Olympus BX51). Photographs of representative colonization classes were taken with an Olympus DP71 camera and processed using the Olympus Cell'B software. The extent of colonization was quantified by assigning inoculation sites to three different classes: I (white), contained a lesion without hyphal colonization outside the necrotic lesion; II (gray), contained a lesion with minimal hyphal growth beyond the lesion; and III (black), contained a spreading lesion with extensive hyphal colonization beyond the lesion. At 4 dai, lesion diameters were determined, as described above.

Extended seed-treatment experiments. Sixty tomato seeds were incubated in $1.5 \mathrm{ml}$ of water (control), BABA $(0.01,0.1$, and $1.0 \mathrm{mM})$ or JA $(0.001,0.01$, and $0.1 \mathrm{mM})$ solution for 7 days at $28^{\circ} \mathrm{C}$ in the dark. Germination efficiency was determined on the basis of the average percentage of germination at 3, 4, and 7 days after the start of imbibition. Ten fully germinated seedlings per selected treatment (control, $1 \mathrm{mM}$ BABA, or $0.1 \mathrm{mM} \mathrm{JA}$ ) were planted individually into 200-ml pots, containing Scotts Levington M3 soil (Everris). Plants were cultivated in controlled environment chambers $(16 \mathrm{~h}$ of light, $150 \mu \mathrm{M} \mathrm{m}^{2} \mathrm{~s}^{-1}$, at $26^{\circ} \mathrm{C}$ and $8 \mathrm{~h}$ of darkness at $21^{\circ} \mathrm{C}$ and approximately $65 \% \mathrm{RH}$ ) for 3 weeks before challenge inoculation with $B$. cinerea. Five plants per treatment were used for inoculations with B. cinerea. Extended seed-treatment experiments were repeated once with similar results.

Seed coating experiments. Coating solutions were prepared by stirring $2 \mathrm{~g}$ of carboxymethyl cellulose (CMC) sodium salt (Sigma catalog number C5013) in $100 \mathrm{ml}$ of $1 \mathrm{mM}$ BABA or $0.1 \mathrm{mM}$ JA. Batches of 12 seeds were immersed into $1 \mathrm{ml}$ of the coating solution (Koistinen 1993). Coated seeds were dried at room temperature for 2 days. Seed were planted individually into $200-\mathrm{ml}$ pots containing Scotts Levington M3 soil (Everris). Plants were cultivated under greenhouse conditions ( $12 \mathrm{~h}$ of light, $>150 \mu \mathrm{M} \mathrm{m}^{2} \mathrm{~s}^{-1}$, at $25^{\circ} \mathrm{C}$ and $12 \mathrm{~h}$ of darkness at $21^{\circ} \mathrm{C}$ ) for 3.5 weeks. Experiments were carried out between October and December 2012 in Sheffield, UK and supplemental light was obtained from sodium vapor lamps. The average percentage of seed germination was determined immediately 
after seed coating (to verify lack of germination by the coating procedure), as well as 7 and 14 days after planting in the soil $(n=12)$. Analysis of RGR and induced resistance to $B$. cinerea were carried out with eight plants per treatment. After 3.5 weeks of growth, plants were challenged with $B$. cinerea. Seed coating experiments were repeated once, with similar results.

Seedling treatments of soil-grown plants. Soil-based experiments were initially grown in 60-ml pots containing Scotts Levington M3 soil (Everris) and cultivated in controlled-environment chambers ( $16 \mathrm{~h}$ of light, $150 \mu \mathrm{M} \mathrm{m}^{2} \mathrm{~s}^{-1}$, at $26^{\circ} \mathrm{C}$ and $8 \mathrm{~h}$ of darkness at $21^{\circ} \mathrm{C}$ and approximately $\left.65 \% \mathrm{RH}\right)$. Seven-day-old seedlings were treated with water (control), BABA, or JA to a final concentration in the growth substrate of $0.1,1$, or $10 \mathrm{mM} \mathrm{BABA}$ or $0.01,0.1$, or $1 \mathrm{mM} \mathrm{JA}$. Chemical treatments were performed by injecting the soil with a $10 \times$ concentrated solution at a volume that was $10 \times$ lower than the pot volume (i.e., $6 \mathrm{ml}$ of solution into $60-\mathrm{ml}$ pots). One week after chemical treatment, roots from 10 plants were gently washed under running tap water, after which seedlings were transplanted into 200-ml pots with untreated soil. After transplantation, plants were grown for 2 weeks until challenge inoculation with $B$. cinerea. Analysis of RGR and induced resistance to $B$. cinerea were carried out with 10 plants per treatment. All soil-based seedling treatment experiments were repeated once, with similar results.

Seedling treatment experiments with hydroponically grown plants. Plants were grown under greenhouse conditions $(12 \mathrm{~h}$ of light, $>150 \mu \mathrm{M} \mathrm{m}^{2} \mathrm{~s}^{-1}$, at $25^{\circ} \mathrm{C}$ and $12 \mathrm{~h}$ of darkness at $21^{\circ} \mathrm{C}$ ) between February and April 2013 in Sheffield, UK with supplemental light from sodium vapor lamps. Seedlings were initially grown in water-soaked 2.5 -cm rock-wool cubes (Growell 0848). One-week-old seedlings were treated with water, BABA, and JA to a final concentration in the rock-wool substrate of $0.1,0.5$, or $1 \mathrm{mM}$ for BABA or $0.01,0.05$, or $0.25 \mathrm{mM}$ for JA. One week after treatment, roots from 10 plants were cleaned by washing the $2.5-\mathrm{cm}$ rock-wool cubes under running tap water, after which the cubes were placed into larger, $10-\mathrm{cm}$ rock-wool cubes. Plants were grown for 2 weeks
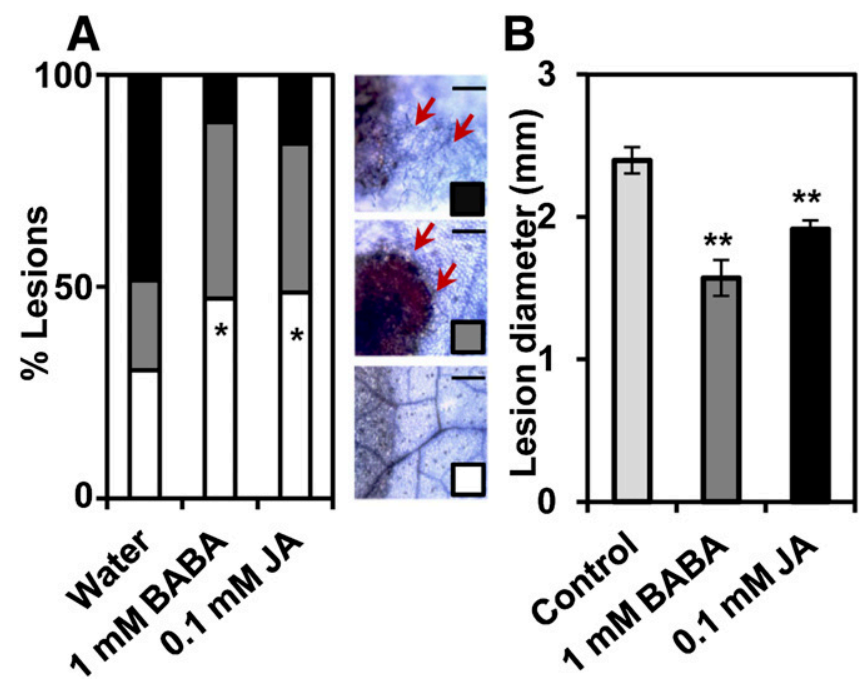

Fig. 1. Validation of a detached-leaf assay for quantification of chemically induced resistance in tomato against Botrytis cinerea. Three-week-old plants were soil drenched with water (control), $1 \mathrm{mM} \beta$-aminobutyric acid (BABA), or $0.1 \mathrm{mM}$ jasmonic acid (JA). Five days later, leaves were detached, inoculated with B. cinerea spores, and examined for hyphal colonization and lesion diameter. A, Relative distribution of inoculation sites over three distinct classes of fungal colonization. Inoculation sites (30 to 40) from 10 plants per treatment were collected from 2 to 3 days after inoculation for trypan-blue staining and examined by light microscopy. Insets show representative examples of each colonization class; red arrows indicate hyphal colonization. Scale bars represent $1 \mathrm{~mm}$. Asterisks indicate a statistically significant difference in colonization compared with watertreated control plants (Fisher's exact tests, $P<0.05 ; n=30$ to 40). B, Average lesion diameters (in millimeters; \pm standard error of the mean; $n=10$ ). Double asterisks indicate a statistically significant difference compared with control-treated plants ( $t$ tests, $P<0.01$ ). and watered by a fully automated nutrient supply system (Vita Link Max Grow; Growell), according to the manufacturer's specifications (three irrigations of $1 \mathrm{~min}$ each, providing a total of $200 \mathrm{ml}$ of full-strength nutrient solution per plant per day). Ten plants per treatment were used for RGR analysis. For the induced resistance assays, only plants pretreated with $0.1 \mathrm{mM} \mathrm{BABA}$ or $0.01 \mathrm{mM} \mathrm{JA}$ were inoculated with $B$. cinerea. Seven individual plants per treatment were used for analysis of the induced resistance. Experiments with hydroponically grown plants were repeated once, with similar results.

AMF experiments. Experiments to determine root colonization by AMF were carried out in a greenhouse with day and night lengths of 16 and $8 \mathrm{~h}$ and temperatures at 26 and $21^{\circ} \mathrm{C}$, respectively. The experiments were carried out between April and July 2014 in Flakkebjerg, Denmark, and sodium vapor lamps at $80 \mu \mathrm{M} \mathrm{m}^{2} \mathrm{~s}^{-1}$ were used as supplementary light on days naturally shorter than $16 \mathrm{~h}$. Growth substrate consisted of a soil and sand mixture (ratio 1:1) with low plant-available $\mathrm{P}(12 \mathrm{ppm})$. Growth substrate was heat sterilized at $80^{\circ} \mathrm{C}$ for $48 \mathrm{~h}$, which was sufficient to prevent AMF colonization from soil-endemic AMF inoculum (data not shown). After sterilization, basic nutrients were mixed into the growth substrate $(42.9 \mathrm{mg}$ of $\mathrm{NH}_{4} \mathrm{NO}_{3}, 37.5 \mathrm{mg}$ of $\mathrm{K}_{2} \mathrm{SO}_{4}, 37.5 \mathrm{mg}$ of $\mathrm{CaCl}_{2} \mathrm{O} \cdot 2 \mathrm{H}_{2} \mathrm{O}, 1.05 \mathrm{mg}$ of $\mathrm{CuSO}_{4}, 2.7 \mathrm{mg}$ of $\mathrm{ZnSO}_{4}, 5.25 \mathrm{mg}$ of $\mathrm{MnSO}_{4}, 0.195 \mathrm{mg}$ of $\mathrm{CoSO}_{4}$, $22.5 \mathrm{mg}$ of $\mathrm{MgSO}_{4}$, and $0.09 \mathrm{mg}$ of $\mathrm{NaMoO}_{4}$ per kilogram of soil) and water-holding capacity was set and maintained at $65 \%$ during the experiments. CMC seed coatings containing water (control) or BABA at $0.1,1.0$, or $10 \mathrm{mM}$ and were carried out as described above. Plants were fertilized with $\mathrm{NH}_{4} \mathrm{NO}_{3}$ weekly. AMF inoculum was provided by Symbiom Ltd. and contained a mixture of five AMF species (Rhizophagus irregularis BEG140, R. irregularis, Funneliformis mosseae BEG95, F. geosporum, and Claroideoglomus claroideum) in a carrier of expanded clay. The inoculum was mixed homogenously into the growth substrate to $10 \%$ of the volume. For the control nonmycorrhizal treatment, the growth substrate received an equal amount of the expanded clay carrier to provide the same physical and chemical growth substrate conditions. For seedling treatments, 7-day-old plants were root drenched with water (control), BABA, or JA to a final concentration in the growth substrate of 0.5 or $0.05 \mathrm{mM} \mathrm{BABA}$ or JA, respectively. AMF colonization was analyzed at 42 days after sowing. Roots were washed, cut into $0.5-\mathrm{cm}$ pieces, and mixed, and random samples of approximately $1 \mathrm{~g}$ of roots from each plant were harvested. These samples were cleared in $10 \% \mathrm{KOH}$ and stained in 5\% ink-blue and AMF colonization was analyzed by microscopy using a lineintersect method, as described previously (Vierheilig et al. 1998). Approximately $1 \mathrm{~g}$ of randomly sampled roots from each replicate plant was microscopically scored for infection, using 200 different viewpoints. Colonization was quantified as the mean percentage of root colonization per plant ( \pm SEM; $n=4$ ).

\section{Results}

Validation of a detached-leaf assay for quantification of chemically induced resistance. To examine whether a previously described detached-leaf assay (Audenaert et al. 2002; Asselbergh et al. 2007; Luna et al. 2014b; Worrall et al. 2012) is suitable for quantifying chemically induced resistance under our growth conditions, 3-week-old tomato plants were soil drenched with water (control), $1 \mathrm{mM} \mathrm{BABA,} \mathrm{or} 0.1 \mathrm{mM}$ JA. Five days later, leaves were detached and inoculated with $B$. cinerea. Induced resistance was quantified on the basis of two parameters: fungal colonization in trypan-blue stained leaves and lesion diameters. BABA- and JAtreated plants showed a statistically significant reduction in hyphal colonization compared with control plants (Fig. 1A; Fisher's exact tests, $P=0.003$ and $P=0.015$, respectively). This reduction in fungal colonization was proportional to a statistically significant reduction in lesion diameter (Fig. $1 \mathrm{~B} ; t$ tests, $P<0.001$ and $P=$ 0.001 , respectively). Hence, lesion diameters are a reliable parameter to quantify reduced $B$. cinerea colonization upon chemical induction of disease resistance. Therefore, subsequent experiments to test durability and effectiveness of chemically induced resistance were based on nondestructive scoring of lesion diameters. 
Effects of extended seed incubation in BABA- or JA-containing solutions. Analysis of seed germination after a 7-day incubation period in solutions containing $\mathrm{BABA}(0.01,0.1$, and $1 \mathrm{mM})$ or JA $(0.001,0.01$, and $0.1 \mathrm{mM}$ ) revealed no negative impacts on seed viability (Fig. 2A). In fact, incubation of seeds in $0.01 \mathrm{mM}$ BABA, $0.1 \mathrm{mM}$ BABA, $0.001 \mathrm{mM}$ JA, or $0.01 \mathrm{mM}$ JA promoted seed germination after 3 and 4 days of imbibition (Fisher's exact tests, $P<0.001$ ). To ensure maximum effectiveness in terms of induced resistance, we used $1 \mathrm{mMBABA}$ and $0.1 \mathrm{mM} \mathrm{JA}$ in subsequent experiments. Analysis of seedling height between the second and third week after chemical treatment did not reveal reductions in RGR by either treatment (Fig. 2B; $t$ tests, $P=0.604$ and 0.830 , respectively). However, 4-week-old plants from BABA- and JA-treated seed showed a statistically significant reduction in lesion diameter after inoculation with $B$. cinerea (Fig. 2C; $t$ tests, $P=0.049$ and 0.038 , respectively).

Effects of seed coating with BABA and JA. To examine whether the effects of extended seed treatment could be mimicked by slowrelease seed coating, we treated seeds with $\mathrm{CMC}$, supplemented with water (control), $1 \mathrm{mM} \mathrm{BABA,} \mathrm{or} 0.1 \mathrm{mM} \mathrm{JA}$. Coating of seed with
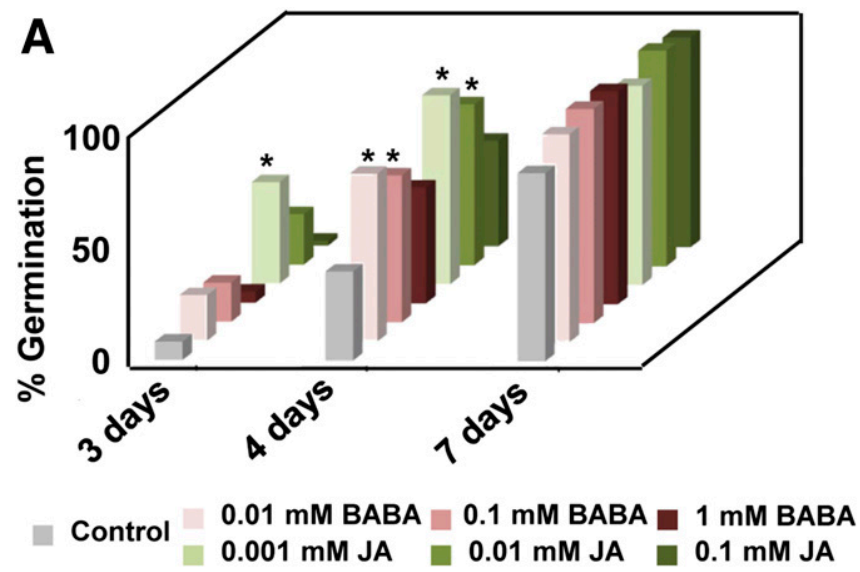

B
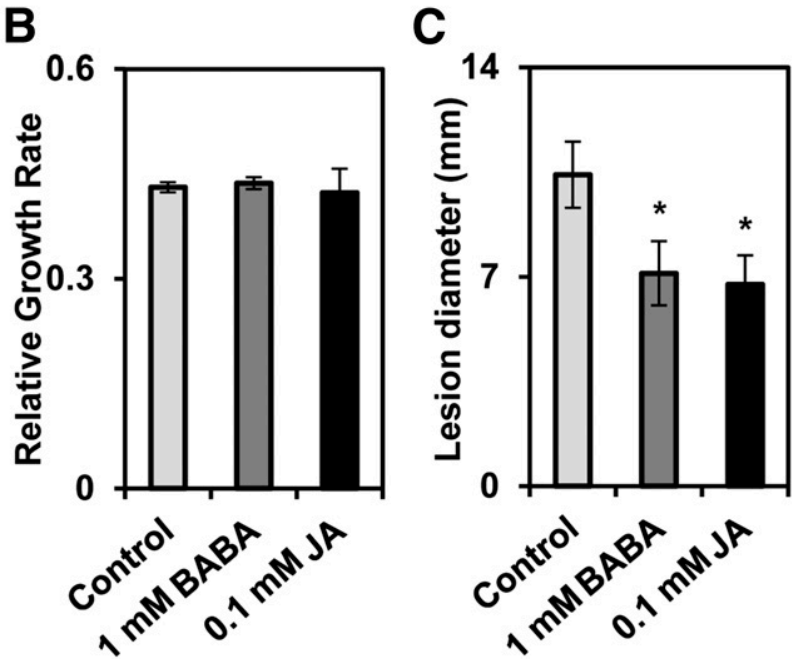

Fig. 2. Effects of extended seed treatment with $\beta$-aminobutyric acid (BABA) and jasmonic acid (JA) on soil-grown tomato. Seed were allowed to germinate for 7 days in water (control) or increasing concentrations of BABA or JA. Seedlings were then transplanted to soil and grown for 3 weeks until challenge inoculation with Botrytis cinerea. A, Percentages of germinated seeds after 3,4 and 7 days of incubation in water (control), BABA, or JA at the indicated concentrations. Asterisks indicate statistically significant differences in germination efficiency compared with control-treated seeds (Fisher's exact test, $P<0.01, n=60$ ); B, Average relative growth rates (in centimeters per centimeter per week; \pm standard error of the mean [SEM]) of plants between 2 and 3 weeks after chemical treatments. No statistically significant differences were detected between treatments ( $t$ test, $\alpha=0.05 ; n=10$ ). C, Average lesion diameters (in millimeters; \pm SEM) at 4 days after inoculation of 4-week-old plants with $B$. cinerea. Asterisks indicate statistically significant differences compared with control-treated plants ( $t$ test, $P<0.05 ; n=5$ ).
BABA or JA did not alter seed germination efficiency (Fig. 3A; Fisher's exact tests, $P>0.05$ ), nor did it lead to reductions in RGR between the second and third week after planting (Fig. 3B; $t$ tests, $P=0.251$ and 0.838 , respectively). Seed coating with JA had no effect on disease lesion diameters by $B$. cinerea in 4 week-old plants (Fig. 3C; $t$ test, $P=0.991$ ). However, plants from BABA-coated seeds developed significantly smaller lesions after $B$. cinerea inoculation in comparison with plants from control-coated seed (Fig. 3C; $t$ test, $P=0.005$ ).

Effects of seedling treatments with BABA or JA on soilgrown plants. To investigate the effects of root treatment of tomato seedlings, 1-week-old seedlings were soil drenched with increasing concentrations of $\mathrm{BABA}$ or $\mathrm{JA}$, and transplanted to untreated soil 1 week later. Soil drenching with the two highest concentrations of $10 \mathrm{mM}$ BABA and $1 \mathrm{mM}$ JA completely abolished plant growth in the following week and was partially lethal (50 and $70 \%$, respectively), which prevented further assessment of disease resistance. Intermediate concentrations of $1 \mathrm{mM}$ BABA and $0.1 \mathrm{mM}$ JA reduced average plant growth to statistically significant levels (Fig. 4A; $t$ tests, $P=0.001$ and 0.034 , respectively), whereas

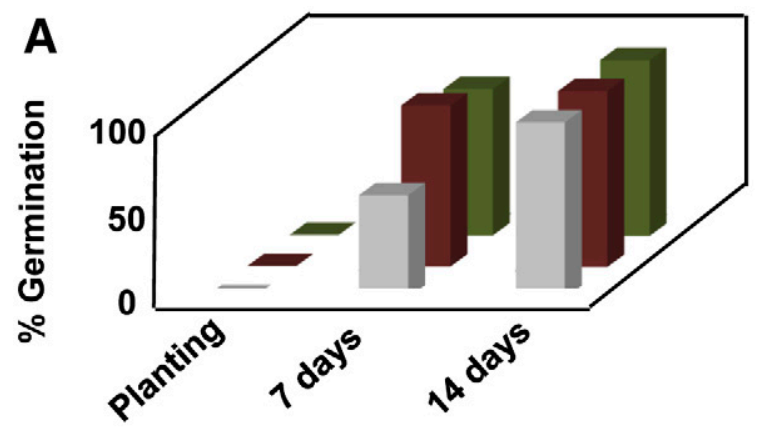

Control $1 \mathrm{mM}$ BABA $=0.1 \mathrm{mM}$ JA

B

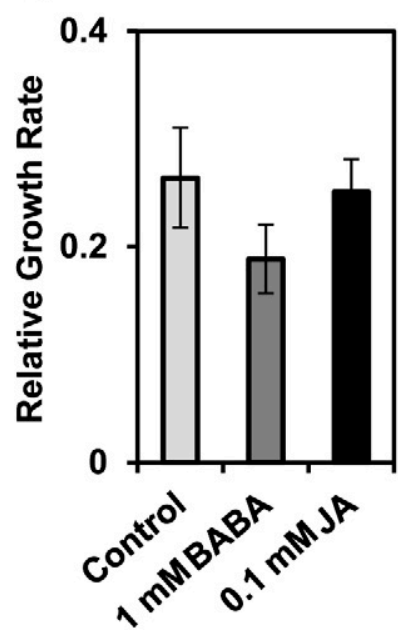

C

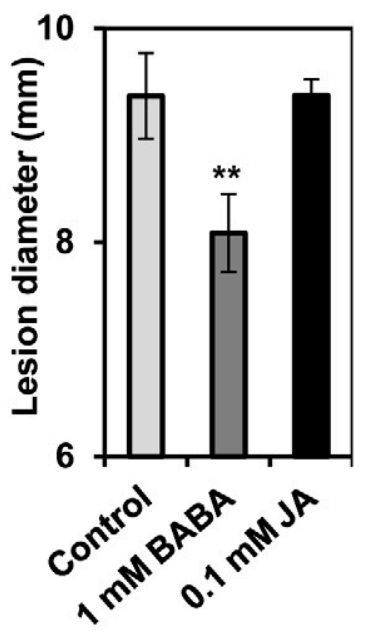

Fig. 3. Effects of seed coating with $\beta$-aminobutyric acid (BABA) and jasmonic acid (JA) on soil-grown tomato. Seed were coated in $2 \%$ carboxymethyl cellulose (CMC) solution containing water (control), $1 \mathrm{mM} \mathrm{BABA}$, or $0.1 \mathrm{mM}$ JA. Two days later, seeds were planted in soil and plants were grown for 3.5 weeks until challenge inoculation with Botrytis cinerea. A, Percentages of germinated seeds at 0,7 , and 14 days after planting. No statistically significant differences in germination efficiency were detected between treatments (Fisher's exact tests, $\alpha=0.05 ; n=12$ ). $\mathbf{B}$, Average relative growth rates (in centimeters per centimeter per week; \pm standard error of the mean [SEM]) between 2 and 3 weeks after planting of coated seed. No statistically significant differences were detected between treatments ( $t$ tests, $\alpha=0.05 ; n=8$ ). C, Average lesion diameters (in millimeters; \pm SEM) at 2 days after inoculation of 4 -week-old plants with $B$. cinerea. Seeds were coated with CMC containing water (control), $1 \mathrm{mM} \mathrm{BABA}$, or $0.1 \mathrm{mM} \mathrm{JA}$. Double asterisk indicates a statistically significant difference compared with controltreated plants ( $t$ tests, $P<0.01 ; n=8$ ). 
the lowest concentrations of $0.1 \mathrm{mM}$ BABA and $0.01 \mathrm{mM} \mathrm{JA}$ had no statistically significant effects on plant growth (Fig. 4A; $t$ tests, $P=0.565$ and 0.321 , respectively). Seedling treatment with the intermediate BABA concentration of $1 \mathrm{mM}$, as well as the lowest and intermediate JA concentration of 0.01 and $0.1 \mathrm{mM}$, respectively, reduced average lesion diameters after inoculation of 4-week-old plants with $B$. cinerea. These effects were statistically significant in comparison with plants grown from control-treated seedlings (Fig. 4B; $t$ tests, $P=0.001,0.047$, and 0.002, respectively).

Effects of seedling treatments with BABA or JA on hydroponically grown plants. Because commercial tomato production is often based on hydroponic growth systems in greenhouses, we tested the effectiveness of applying BABA and JA to roots of rock-wool-cultivated tomato. Treatment of 1-week-old seedlings with $0.5 \mathrm{mM}$ BABA, $1 \mathrm{mM}$ BABA, or $0.25 \mathrm{mM}$ JA resulted in statistically significant levels
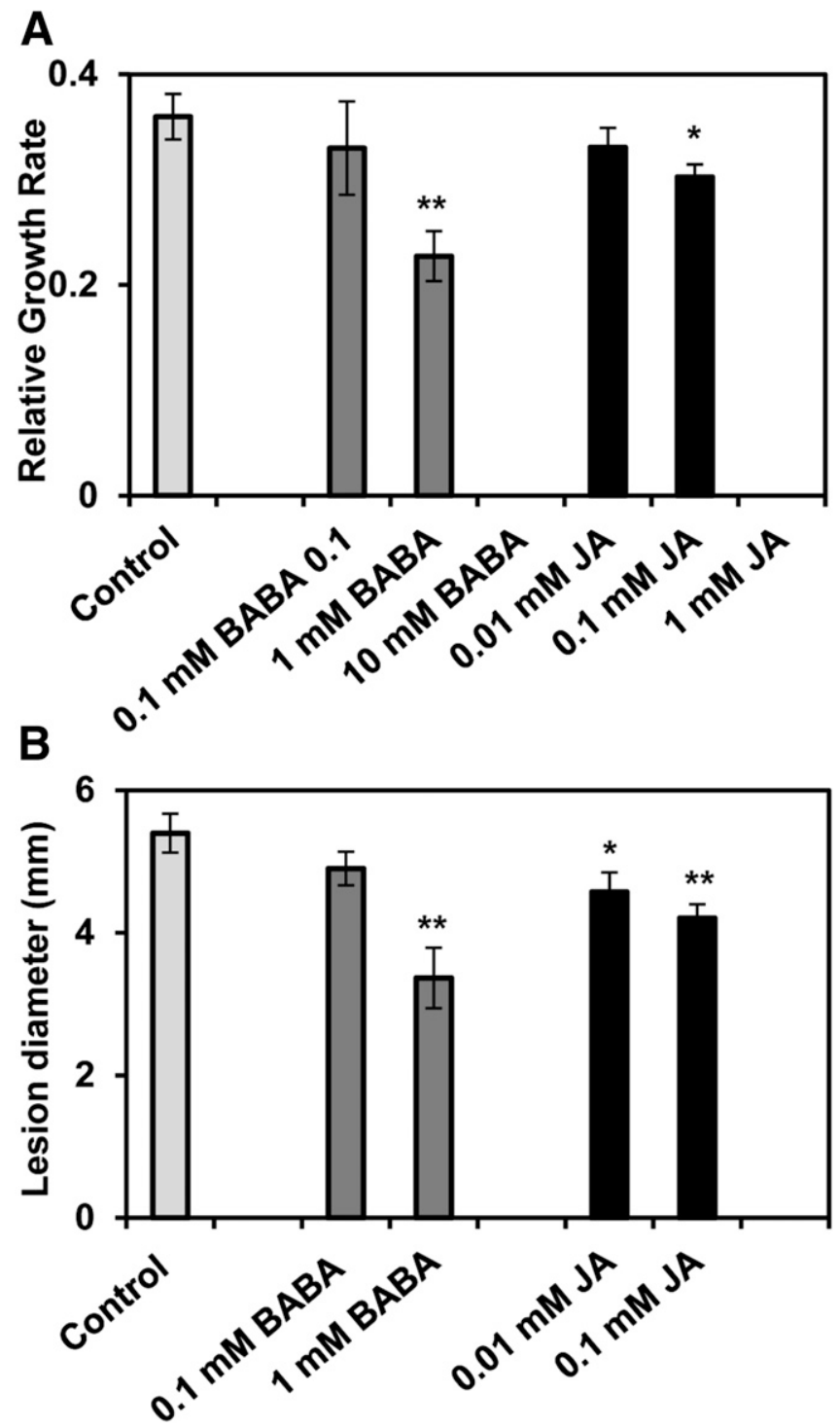

Fig. 4. Effect of seedling treatment with $\beta$-aminobutyric acid (BABA) and jasmonic acid $(\mathrm{JA})$ on soil-grown tomato. One-week-old seedlings were soil drenched with water (control) or increasing concentrations of BABA $(0.1,1$, and $10 \mathrm{mM})$ or JA $(0.01,0.1$, and $1 \mathrm{mM})$. One week later, seedlings were transplanted to untreated soil and cultivated for two additional weeks before challenge inoculation with Botrytis cinerea. A, Average relative growth rates (in centimeters per centimeter per week; \pm standard error of the mean [SEM]) in 2- to 3-week-old plants. Asterisks indicate statistically significant differences compared with control-treated plants ( $t$ tests, single asterisk: $0.05<P<0.01$; double asterisk: $P<0.01 ; n=10$ ). B, Average lesion diameters (in millimeters; \pm SEM) at 4 days after inoculation of 4-week-old plants with $B$. cinerea. Asterisks indicate statistically significant differences compared with control-treated plants ( $t$ tests, single asterisk: $0.05<P<$ 0.01; double asterisk: $P<0.01 ; n=10$ ). of growth repression between the second and third week after planting (Fig. 5A; $t$ tests, $P=0.042,0.005$, and 0.031 , respectively). In comparison with soil-grown plants (Fig. 4A), root treatment of 1-week-old seedlings with $1 \mathrm{mM}$ BABA caused more severe growth reduction in hydroponically grown plants (Fig. 5A). Nevertheless, root drenching with relatively low concentrations of BABA $(0.1 \mathrm{mM})$ or JA $(0.1$ and $0.01 \mathrm{mM}$ ) did not affect plant growth (Fig. $5 \mathrm{~A} ; t$ tests, $P=0.783$, 0.377 , and 0.085 , respectively). Furthermore, the relatively low and stress-free concentrations of $0.1 \mathrm{mM} \mathrm{BABA}$ and $0.01 \mathrm{mM}$ JA reduced average lesion diameters by $B$. cinerea after inoculation of 4-week-old plants (Fig. 5B; $t$ tests, $P=0.024$ and $P<0.001$, respectively).

Nontarget effects of BABA and JA on root colonization by AMF. To evaluate nontarget effects of BABA and JA on beneficial soil microbes, we determined impacts of seed coating and soil

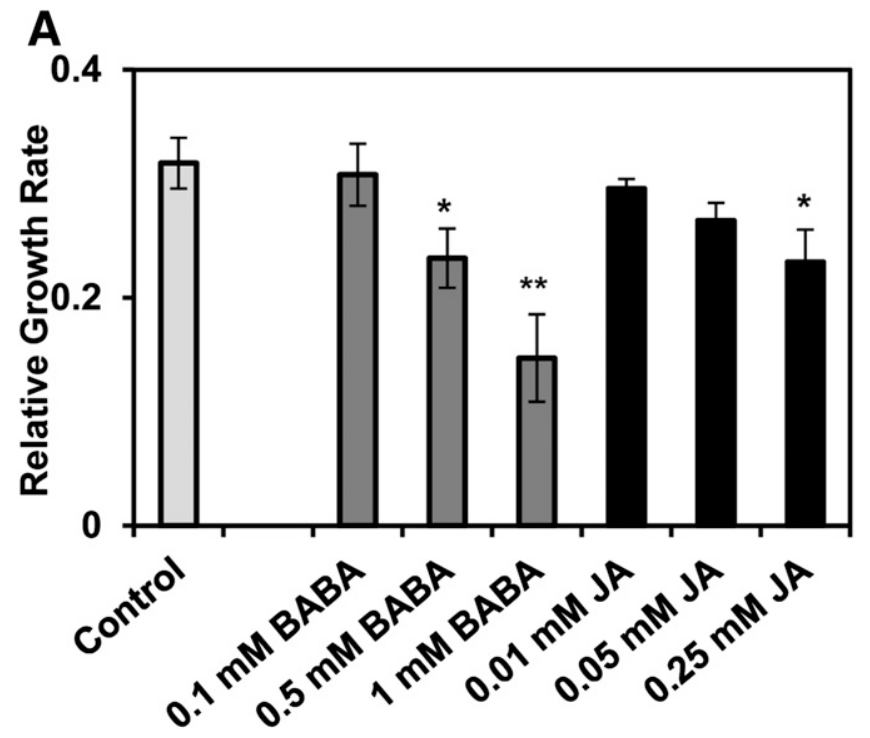

B

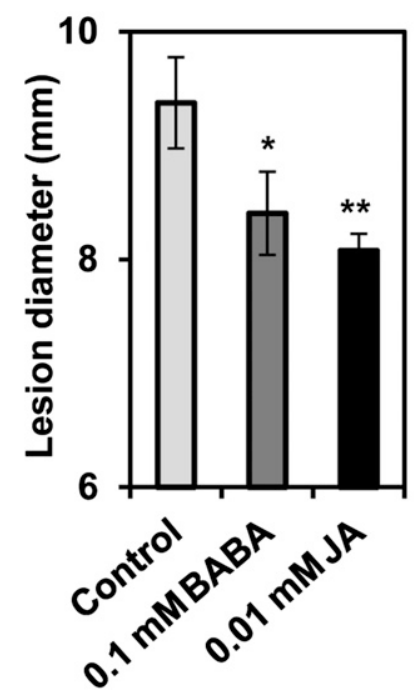

Fig. 5. Effects of seedling treatment with $\beta$-aminobutyric acid (BABA) and jasmonic acid (JA) on hydroponically grown tomato. One-week-old seedlings were soil drenched with water (control) or different concentrations of BABA or JA. One week after root treatment, small rock-wool cubes were placed into a larger $10 \mathrm{~cm}$ rock wool cubes, and cultivated for 2 weeks before challenge inoculation with Botrytis cinerea. A, Average relative growth rates (in centimeters per centimeter per week; \pm standard error of the mean [SEM]) in 2- to 3-week-old plants. Asterisks indicate statistically significant differences compared with control-treated plants ( $t$ tests, single asterisk: $0.05<P<0.01$; double asterisk: $P<0.01 ; n=10$ ). B, Average lesion diameters (in millimeters; \pm SEM) at 4 days after inoculation of 4 -week-old plants with $B$. cinerea. Asterisks indicate statistically significant differences compared with control-treated plants ( $t$ tests, single asterisk: $0.05<P<0.01$; double asterisk: $P<0.01 ; n=7)$. 
drenching on root colonization by AMF. CMC coating with increasing BABA concentrations $(0.1,1$, and $10 \mathrm{mM}$ ) enhanced AMF colonization of 6-week-old tomato plants slightly, although this trend was not statistically significant (Fig. 6A; $t$ tests, $P=0.10,0.41$, and 0.16 , respectively). Furthermore, seedling treatment with the relatively high concentration of $0.5 \mathrm{mM}$ BABA had no effect on AMF colonization in 6-week-old plants (Fig. 6B; $t$ test, $P=0.93$ ). Seedlings treated with the relatively high concentration of $0.05 \mathrm{mM} \mathrm{JA}$ weakly reduced AMF colonization in comparison with plants grown from control-treated seedlings, but this effect was not statistically significant (Fig. 6B; $t$ test, $P=0.36$ ).

\section{Discussion}

Seed treatment with resistance-inducing chemicals has emerged as a novel strategy to protect tomato against disease and herbivory (Paudel et al. 2014; Worrall et al. 2012). However, similar MeJA seed treatment of Money-Maker tomato failed to induce resistance against T. urticae (Smart et al. 2013). Although it is possible that genetic variation in seed coat permeability explains cultivarspecific effectiveness of seed treatment, extended incubation of germinating Money-Maker seed in BABA- or JA-containing solutions induced durable resistance to $B$. cinerea without adverse effects on plant growth (Fig. 2B and C). Moreover, these treatments also accelerated germination (Fig. 2A), suggesting priming effects on seed germination. Thus, extended exposure of germinating seeds to BABA and JA bypasses the limitations of chemical impermeability of the tomato seed coat and increases the effectiveness of induced resistance without negatively affecting seed germination.

To translate the outcome of the extended seed treatment experiments to a commercially practical application, we tested slowrelease seed coatings with BABA and JA. Seed coating is a common technique to improve seed conservation and germination, while protecting the emerging seedling against soil-borne diseases (El-Abyad et al. 1993; Govinden-Soulange and Levantard 2008; Haigh and Barlow 1987). However, a complicating factor with chemical delivery via seed coating is the endosperm, which can act as a barrier against water uptake (Leviatov et al. 1994). For this reason, slowrelease seed coatings have been developed to ensure sufficient uptake of chemicals during seed germination (Taylor et al. 1998).

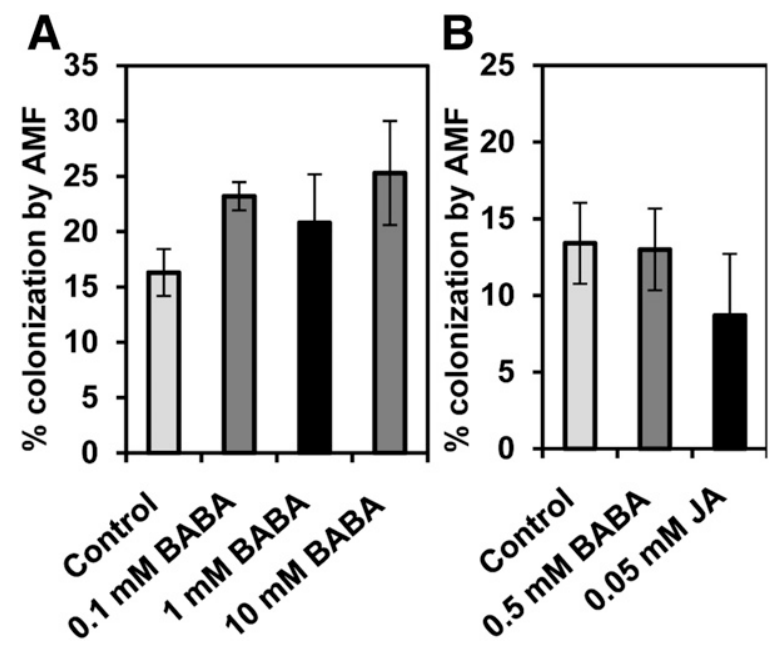

Fig. 6. Effects of $\beta$-aminobutyric acid (BABA) and jasmonic acid (JA) on root colonization by arbuscular mycorrhizal fungi (AMF) in soil-grown tomato. Plants were grown in soil supplemented with a mixture of five AMF species. A, Average percentages ( \pm standard error of the mean [SEM]) of root colonization by AMF in 6 -week-old plants that had been grown from carboxymethyl-cellulose-coated seed, containing water or increasing concentrations of BABA. No statistically significant differences in AMF colonization were found between treatments ( $t$ tests, $\alpha=0.05$; $n=4$ ) B, Average percentages ( \pm SEM) of root colonization by AMF in 6-week-old plants. One-week-old seedlings were soil drenched with water (control), $0.5 \mathrm{mM}$ $\mathrm{BABA}$, or $0.05 \mathrm{mM} \mathrm{JA}$. No statistically significant differences in AMF colonization were found between treatments ( $t$ tests, $\alpha=0.05 ; n=4$ )
$\mathrm{CMC}$ is a cellulose derivative (E466) that is commonly used as a thickener or stabilizer in a wide range of food, nonfood, and pharmaceutical products. CMC is also used as slow-release seed coating to deliver fungicides (Scott 1961). In our experiments, CMC seed coating with $1 \mathrm{mM} \mathrm{BABA}$ induced durable resistance against $B$. cinerea (Fig. 3C), which was not associated with negative effects on seed germination (Fig. 3A) or plant growth (Fig. 3B). Surprisingly, however, CMC coating with JA had no effect on disease resistance (Fig. 3C), even though extended seed germination in JA solution induced resistance to B. cinerea (Fig. 2C). This unexpected result may be due to the physiochemical properties of BABA and JA: whereas BABA is a highly water-soluble compound, JA is a derivative of the fatty acid linolenic acid and, therefore, more lipophilic. Accordingly, it is possible that alternative slow-release matrices offer better results with JA.

Root-drench treatment of 1-week-old seedlings with BABA or JA induced durable resistance in 4-week-old plants (Figs. 4B and 5B). At relatively high concentrations of these chemicals, the induced resistance was associated with repression of plant growth (Figs. 4A and 5A), which was particularly pronounced in hydroponically cultivated plants after treatment with BABA. The costs of induced resistance have previously been linked to reductions in plant growth and seed production (Heil 2007; Louws et al. 2001; van Hulten et al. 2006). However, in the context of agricultural exploitation of plant defense activators, indirect costs of induced resistance on beneficial ecological interactions are often overlooked (Heil 2002), even though they are increasingly considered as a means to intensify sustainable agriculture (Power 2010). AMF can reduce fertilizer input and enhance stress resilience in crops, making them attractive for exploitation in sustainable agriculture (Cameron et al. 2013; Evelin et al. 2009; Pozo et al. 2013). Our experiments revealed no negative impacts of BABA and JA on AMF colonization, despite the relatively high chemical concentrations used in these experiments (Fig. 6). Therefore, we conclude that BABA- and JA-induced resistance does not pose costs on AMF colonization in Money-Maker tomato.

Our study has shown that resistance-inducing chemicals can offer durable protection of tomato under commercial growth conditions. Whereas seed coating with BABA can be used in soil-based cultivation systems of tomato, hydroponic growth systems in greenhouses lend themselves well for controlled applications of $\mathrm{BABA}$ or JA to seedlings via the water supply system. Furthermore, none of the application methods to soil-grown tomato affected AMF colonization negatively, indicating potential for integrated strategies combining chemically induced resistance with AMF. However, despite the fact that the protective effects of $\mathrm{BABA}$ and $\mathrm{JA}$ could be confirmed under commercial greenhouse conditions (Figs. 3C and 5B), the quantification of disease resistance was based on a detached-leaf assay (Fig. 1). Although this assay is sensitive for detecting subtle changes in disease resistance by ensuring optimal pathogen virulence and minimal environmental variation, the inoculations are performed with relatively high spore densities, which rarely occur under commercial growth conditions. Therefore, the protective effects of the resistanceinducing chemicals on natural outbreaks of $B$. cinerea remain to be investigated. It will also be important to examine whether chemically induced resistance protects tomato plants until harvest, and how far the combination of chemical treatment and disease pressure affects plant growth and fruit set. In this context, we emphasize that our study is of translational value: its outcome offers useful information for large-scale trials that aim to determine whether chemically induced resistance entails a commercially viable alternative for reducing fungicide use in the tomato industry.

\section{Acknowledgments}

The research was supported by a consolidator grant from the European Research Council (ERC; number 309944-Prime-A-Plant) to J. Ton, a Research Leadership Award from the Leverhulme Trust (number RL-2012-042) to J. Ton, and European Union 7th Framework Programme (FP7/2007-2013; number 265865-PURE) to J. Ton and S. Ravnskov. 


\section{Literature Cited}

Asselbergh, B., Curvers, K., França, S. C., Audenaert, K., Vuylsteke, M., Van Breusegem, F., and Höfte, M. 2007. Resistance to Botrytis cinerea in sitiens, an abscisic acid-deficient tomato mutant, involves timely production of hydrogen peroxide and cell wall modifications in the epidermis. Plant Physiol. 144:1863-1877.

Audenaert, K., De Meyer, G. B., and Hofte, M. M. 2002. Abscisic acid determines basal susceptibility of tomato to Botrytis cinerea and suppresses salicylic aciddependent signaling mechanisms. Plant Physiol. 128:491-501.

Bautista-Baños, S., Hernández-Lauzardo, A. N., Velázquez-Del Valle, M. G., Hernández-López, M., Ait Barka, E., Bosquez-Molina, E., and Wilson, C. L. 2006. Chitosan as a potential natural compound to control pre and postharvest diseases of horticultural commodities. Crop Prot. 25:108-118.

Brévault, T., and Bouyer, J. 2014. From integrated to system-wide pest management: Challenges for sustainable agriculture. Population (Paris) 69:212-213.

Cameron, D. D., Neal, A. L., Van Wees, S. C. M., and Ton, J. 2013. Mycorrhizainduced resistance: More than the sum of its parts? Trends Plant Sci. 18:539-545.

Cohen, Y. 2002. Beta-aminobutyric acid-induced resistance against plant pathogens. Plant Dis. 86:448-457.

Cohen, Y., Niderman, T., Mosinger, E., and Fluhr, R. 1994. [beta]-Aminobutyric acid induces the accumulation of pathogenesis-related proteins in tomato (Lycopersicon esculentum L.) plants and resistance to late blight infection caused by Phytophthora infestans. Plant Physiol. 104:59-66.

de Román, M., Fernández, I., Wyatt, T., Sahrawy, M., Heil, M., and Pozo, M. J. 2011. Elicitation of foliar resistance mechanisms transiently impairs root association with arbuscular mycorrhizal fungi. J. Ecol. 99:36-45.

Durrant, W. E., and Dong, X. 2004. Systemic acquired resistance. Annu. Rev. Phytopathol. 42:185-209.

El-Abyad, M. S., El-Sayed, M. A., El-Shanshoury, A. R., and El-Sabbagh, S. M. 1993. Towards the biological control of fungal and bacterial diseases of tomato using antagonistic Streptomyces spp. Plant Soil 149:185-195.

Elad, Y., Williamson, B., Tudzynski, P., and Delen, N. 2007. Botrytis spp. and Diseases They Cause in Agricultural Systems - An Introduction. Pages 1-8 in: Botrytis: Biology, Pathology and Control. Y. Elad, B. Williamson, P. Tudzynski, and N. Delen, eds. Springer, Dordrecht, The Netherlands.

El Oirdi, M., El Rahman, T. A., Rigano, L., El Hadrami, A., Rodriguez, M. C., Daayf, F., Vojnov, A., and Bouarab, K. 2011. Botrytis cinerea manipulates the antagonistic effects between immune pathways to promote disease development in tomato. Plant Cell 23:2405-2421.

Evelin, H., Kapoor, R., and Giri, B. 2009. Arbuscular mycorrhizal fungi in alleviation of salt stress: A review. Ann. Bot. (Lond.) 104:1263-1280.

FAOSTAT. 2014. Food and Agricultural commodities production. Commodities by regions. Online publication. http://faostat3.fao.org/browse/rankings/ commodities_by_regions/E

Faretra, F., and Pollastro, S. 1991. Genetic basis of resistance to benzimidazole and dicarboximide fungicides in Botryotinia fuckeliana (Botrytis cinerea). Mycol. Res. 95:943-951.

Flors, V., Miralles, C., Gonzalez-Bosch, C., Carda, M., and Garcia-Agustin, P. 2003. Three novel synthetic amides of adipic acid protect Capsicum anuum plants against the necrotrophic pathogen Alternaria solani. Physiol. Mol. Plant Pathol. 63:151-158.

Fujimoto, T., Tomitaka, Y., Abe, H., Tsuda, S., Futai, K., and Mizukubo, T. 2011. Expression profile of jasmonic acid-induced genes and the induced resistance against the root-knot nematode (Meloidogyne incognita) in tomato plants (Solanum lycopersicum) after foliar treatment with methyl jasmonate. J. Plant Physiol. 168:1084-1097.

Govinden-Soulange, J., and Levantard, M. 2008. Comparative studies of seed priming and pelleting on percentage and meantime to germination of seeds of tomato (Lycopersicon esculentum Mill.). Afr. J. Agric. Res. 3:725-731.

Haigh, A., and Barlow, E. 1987. Water relations of tomato seed germination. Funct. Plant Biol. 14:485-492.

Heil, M. 2002. Ecological costs of induced resistance. Curr. Opin. Plant Biol. 5:345-350.

Heil, M. 2007. Trade-offs associated with induced resistance. Pages 157-177 in: Induced Resistance for Plant Defence: A Sustainable Approach to Crop Protection. Blackwell, Oxford.

Iriti, M., and Faoro, F. 2009. Chitosan as a MAMP, searching for a PRR. Plant Signal. Behav. 4:66-68.

Kępczyńska, E., and Król, P. 2012. The phytohormone methyl jasmonate as an activator of induced resistance against the necrotroph Alternaria porri f. sp. solani in tomato plants. J. Plant Interact. 7:307-315.

Koistinen, T. 1993. Compositions and Methods for Coating Seed. Google Patents, http://www.google.com/patents/WO1993025078A1?cl=en
Leviatov, S., Shoseyov, O., and Wolf, S. 1994. Roles of different seed components in controlling tomato seed germination at low temperature. Sci. Hortic. 56: 197-206.

Louws, F. J., Wilson, M., Campbell, H. L., Cuppels, D. A., Jones, J. B., Shoemaker, P. B., Sahin, F., and Miller, S. A. 2001. Field Control of Bacterial Spot and Bacterial Speck of Tomato Using a Plant Activator. Plant Dis. 85:481-488.

Luna, E., Lopez, A., Kooiman, J., and Ton, J. 2014a. Role of NPR1 and KYP in longlasting induced resistance by beta-aminobutyric acid. Front. Plant Sci. 5:184.

Luna, E., van Hulten, M., Zhang, Y., Berkowitz, O., Lopez, A., Petriacq, P., Sellwood, M. A., Chen, B., Burrell, M., Van De Meene, A., Pieterse, C. M., Flors, V., and Ton, J. 2014b. Plant perception of beta-aminobutyric acid is mediated by an aspartyl-tRNA synthetase. Nat. Chem. Biol. 10:450-456.

Mandal, S., Mallick, N., and Mitra, A. 2009. Salicylic acid-induced resistance to Fusarium oxysporum f. sp. lycopersici in tomato. Plant Physiol. Biochem. 47:642-649.

Oka, Y., Cohen, Y., and Spiegel, Y. 1999. Local and systemic induced resistance to the root-knot nematode in tomato by DL- $\beta$-amino-n-butyric acid. Phytopathology 89:1138-1143.

Pastor, V., Luna, E., Mauch-Mani, B., Ton, J., and Flors, V. 2013. Primed plants do not forget. Environ. Exp. Bot. 94:46-56.

Paudel, S., Rajotte, E. G., and Felton, G. W. 2014. Benefits and costs of tomato seed treatment with plant defense elicitors for insect resistance. ArthropodPlant Interact. 8:539-545.

Power, A. G. 2010. Ecosystem services and agriculture: Tradeoffs and synergies. Philos. Trans. R. Soc. B. Biol. Sci. 365:2959-2971.

Pozo, M., Jung, S., Martínez-Medina, A., López-Ráez, J., Azcón-Aguilar, C., and Barea, J.-M. 2013. Root allies: Arbuscular mycorrhizal fungi help plants to cope with biotic Stresses. Pages 289-307 in: Symbiotic Endophytes. R. Aroca, ed. Springer, Berlin, Heidelberg.

Rasmann, S., De Vos, M., Casteel, C. L., Tian, D., Halitschke, R., Sun, J. Y., Agrawal, A. A., Felton, G. W., and Jander, G. 2012. Herbivory in the previous generation primes plants for enhanced insect resistance. Plant Physiol. 158: 854-863.

Scalschi, L., Vicedo, B., Camanes, G., Fernandez-Crespo, E., Lapena, L., Gonzalez-Bosch, C., and Garcia-Agustin, P. 2013. Hexanoic acid is a resistance inducer that protects tomato plants against Pseudomonas syringae by priming the jasmonic acid and salicylic acid pathways. Mol. Plant Pathol. 14:342-355.

Scott, D. 1961. Method for treating seeds, and product of said method. Google Patents, California, http://www.google.com/patents/US2967376

Smart, L., Martin, J., Limpalaër, M., Bruce, T. A., and Pickett, J. 2013. Responses of herbivore and predatory mites to tomato plants exposed to jasmonic acid seed treatment. J. Chem. Ecol. 39:1297-1300.

Sonnemann, I., Finkhaeuser, K., and Wolters, V. 2002. Does induced resistance in plants affect the belowground community? Appl. Soil Ecol. 21:179-185.

Taylor, A. G., Allen, P. S., Bennett, M. A., Bradford, K. J., Burris, J. S., and Misra, M. K. 1998. Seed enhancements. Seed Sci. Res. 8:245-256.

Tosi, L., and Zazzerini, A. 2000. Interactions between Plasmopara helianthi, Glomus mosseae and two plant activators in sunflower plants. Eur. J. Plant Pathol. 106:735-744.

Troisi, M., Gimmillaro, M. P., Mangiapan, S., Pirovano, C., and Pasquini, S. 2012. Penthiopyrad (Fontelis ${ }^{\circledR}$ ), new broad spectrum fungicide: Experiences to control botrytis and sclerotinia on vegetable crops. Pages 559-564 in: Giornate Fitopatologiche. Milano Marittima (RA), Italy.

van Hulten, M., Pelser, M., Van Loon, L., Pieterse, C. M. J., and Ton, J. 2006 Costs and benefits of priming for defense in Arabidopsis. Proc. Natl. Acad. Sci. USA 103:5602-5607.

Vicedo, B., Flors, V., De La, O. L. M., Finiti, I., Kravchuk, Z., Real, M. D., GarciaAgustin, P., and Gonzalez-Bosch, C. 2009. Hexanoic acid-induced resistance against Botrytis cinerea in tomato plants. Mol. Plant-Microbe Interact. 22: 1455-1465.

Vierheilig, H., Coughlan, A. P., Wyss, U., and Piche, Y. 1998. Ink and vinegar, a simple staining technique for arbuscular-mycorrhizal fungi. Appl. Environ. Microbiol. 64:5004-5007.

Walters, D., and Heil, M. 2007. Costs and trade-offs associated with induced resistance. Physiol. Mol. Plant Pathol. 71:3-17.

Walters, D. R., Ratsep, J., and Havis, N. D. 2013. Controlling crop diseases using induced resistance: Challenges for the future. J. Exp. Bot. 64: 1263-1280.

Worrall, D., Holroyd, G. H., Moore, J. P., Glowacz, M., Croft, P., Taylor, J. E., Paul, N. D., and Roberts, M. R. 2012. Treating seeds with activators of plant defence generates long-lasting priming of resistance to pests and pathogens. New Phytol. 193:770-778. 